\title{
Medicación mantenida con inhibidores de la fosfodiesterasa (PDE-5) ¿algo más que tratar la disfunción eréctil?
}

\author{
Vela Navarrete R, García Cardoso JV, López Farre A, Guesada Romero P, Pardo Montero M. \\ Cátedra de Urología de la Universidad Autónoma de Madrid. Servicio de Urología Fundación Jiménez Díaz \\ y Unidad de Investigación Cardiovascular del Hospital Clínico San Carlos. Madrid.
}

Actas Urol Esp. 2007;31(3);179-184

$\mathrm{L}$ a tendencia actual a tratar los pacientes con disfunción eréctil (D.E.) de aparente o evidente etiología vascular, de manera continuada, pautada o crónica, está basada en diferentes argumentos e hipótesis cuya validez, pretendemos analizar, con fines críticos y prospectivos, indagando sobre evidencias confirmadas y proyectos de investigación pendientes, en el siguiente texto. Más concretamente, deseamos documentar si la reciente afirmación de I. Goldstein (European Society for Sexual Medicine. Viena. Diciembre 4 2006) ${ }^{4}$ es definitivamente asumible o no: "El tratamiento crónico con los inhibidores de la PDE-5 debe llegar a ser la norma ya que tal tratamiento mejora la salud eréctil, cardiaca y endotelial”.

Los argumentos básicos que fundamentan la tendencia al tratamiento continuo con los inhibidores de PDE-5 (I-PDE5) son los siguientes: (Tabla 1).

1. La presunción conceptual, generalmente aceptada, de que "un padecimiento crónico necesita un tratamiento crónico". Ciertamente, otras muchas enfermedades crónicas son subsidiarias de tratamientos mantenidos cuyas limitaciones están marcadas por el cumplimiento de objetivos de eficacia a largo plazo, en ausencia de efectos indeseables, también a largo término (tolerancia) y el cumplimiento, por parte de los pacientes, no siempre entusiasmados con la idea de la servidumbre crónica.

2. El argumento patogénico, indisolublemente asociado al nuevo y generalmente aceptado concepto de la disfunción endotelial (DEN) como proceso subyacente de la patología que motiva la D.E. en estos pacientes (Feldman et al $1994^{1}$; Bivalacqua et al $2003^{2}$ ). Confirmada mediante numerosos estudios la excelente respuesta del endotelio peneano a los IPDE-5, con la vasodilatación y erección consecuente, se aventura la hipótesis de que el tratamiento mantenido, motivando erecciones más frecuentes, no necesariamente relacionadas con la actividad sexual (erecciones durante el sueño, ocasionales durante el día) significa una mejor y más frecuente oxigenación del área vascular peneana, mejorando funcionalidad endotelial y soslayando fenómenos degenerativos, tanto cuantitativos como cualitativos (Moreland R.B. 2000³).

Tabla 1

Tratamiento médico de la disfunción eréctil tratamiento continuado vs a demanda; pros y contras

\begin{tabular}{ll}
\hline Tratamiento intermitente o a demanda & Tto. pautado, continuo o crônico \\
\hline La naturaleza intermitente de la actividad sexual & $\begin{array}{l}\text { Mejor respuesta en pacientes con fracaso a demanda (McMahon } \\
\text { 2004-2005). }\end{array}$ \\
$\begin{array}{ll}\text { Superior relación coste-eficacia que con el } \\
\text { tratamiento crónico (McMahon 2006) }\end{array}$ & $\begin{array}{l}\text { Más aceptación o preferencia en algunas series (McMahon 2005) } \\
\text { Más aceptación o preferencia de los pacientes } \\
\text { (Mirone et al 2005) }\end{array}$ \\
$\begin{array}{l}\text { Concepto logístico: enfermedades crónicas necesitan } \\
\text { tratamientos crónicos. }\end{array}$ \\
$\begin{array}{l}\text { Potenciales beneficios endoteliales, peneanos (corpora cavernosa) } \\
\text { del fármaco, dosia y y estrategia (farmacodinamia) }\end{array}$ & $\begin{array}{l}\text { y sistémicos. } \\
\text { Potenciales beneficios sobre función endotelial y salud } \\
\text { cardiovascular. } \\
\text { Potenciales beneficios control hipertensión (casos de hipertensión } \\
\text { asociados con AAgII) }\end{array}$ \\
\hline
\end{tabular}


3. La hipótesis más avanzada y ambiciosa, también fundamentada en la teoría endotelial de la D.E., extiende los beneficios señalados para el endotelio peneano, a todo el árbol vascular, incluyendo de manera prominente el área coronaria. Dicho de otro modo; ¿con el tratamiento continuado estamos haciendo prevención de la enfermedad coronaria?. Más aún: ¿estamos haciendo prevención de otras enfermedades cuya etiología esté fundamentalmente relacionada con disfunción endotelial? (Goldstein 20064).

4. En una visión más específicamente urológica, el uso continuado de los I-PDE5 puede ser útil en problemas de rehabilitación endotelial tras lesiones traumáticas peneanas, vasculares, neurales o mixtas (la más común, la secundaria a la prostatectomía radical, con o sin preservación de las bandeletas neurovasculares ${ }^{5}$ ). También se aventura una función curativa o preventiva, al menos sintomáticamente eficaz, en otros procesos disfuncionales como la sintomatología del prostatismo, subsidiaria de la dinámica del óxido nítrico en algunos de sus sintomas ${ }^{6}$.

En definitiva, un acercamiento crítico a este formidable proyecto terapéutico, de tan extraordinaria amplitud, siempre tomando como referencia la disfunción endotelial, nos obligaría a escrutar evidencias y especulaciones sobre aspectos muy variados que ordenamos del siguiente modo: En primer lugar, sobre las evidencias clínicas y sus métodos de valoración; en segundo lugar, sobre evidencias experimentales. Nos referiremos fundamentalmente a los puntos 1,2 y 3 previamente mencionados.

\section{EVIDENCIAS CLINICAS}

La población inicialmente incluida en estos estudios es la población masculina con D.E. vasculogénica que no respondió al tratamiento a demanda (McMahon 200477). Más tarde, los estudios comparativos (placebo, diferentes dosis, diferentes pautas, etc.) han intentado definir aspectos relacionados con los siguientes objetivos: preferencias de los pacientes; tolerancia; beneficios de fármacos concretos (Sildenafil/Tadanafil/Vardenafil) en función de su farmacodinámica; dosis y pautas; potencial producción de taquifilaxia. (Tabla 2). En relación con la preferencia de los pacientes los resultados, con el mismo fármaco,
Tabla 2

Tratamiento médico de la disfunción eréctil objetivos generales de los ensayos clínicos con tratamiento continuado

¿Preferencia de los pacientes, en general?

¿Tolerancia similar o diferente?

¿Mayor eficacia que el tratamiento a demanda?

¿Mejor farmaco y pauta para conseguir eficacia optima?

¿Eficacia mantenida o taquifilaxia?

pueden variar entre $72 \%$ (McMahon $2005^{8}$ ) o el $42 \%$ (Mirone et al 2005 ${ }^{9}$ ). En nuestro ambiente, los pacientes incluidos en el Estudio Sure prefirieron recibir la medicación en régimen a demanda que pautado $(55,9 \%$ vs $44.1 \%$ p $<0,05)$ (Martín Morales et al 2006).

Disponemos de numerosos trabajos de tratamiento continuado con cada uno de los PDE-5 existentes en el mercado (Tabla 3): De este modo, Desouza et al $2002^{11}$, Sommer y Schulze $(2005)^{12}$, han estudiado sildenafil. Rosano et al $2005^{13}$, Caretta et al 2005 ${ }^{14}$, McMahon 2005, Mirone et al 2005 y Porst et al $2006^{15}$, con Tadalafil. Existe una reciente y amplia revisión de la literatura sobre estos tratamientos (Montorsi et al 2006) ${ }^{16}$ con las siguientes conclusiones generales: La medicación continuada con los PDE-5 como potencial estrategia curativa de la D.E y de la disfunción endotelial es un proyecto intelectualmente interesante y terapéuticamente atractivo (Hellstrom y Kendirci 2005 ${ }^{17}$ ); Los datos actuales justifican la hipótesis de que la estimulación endotelial mantenida puede prevenir, e incluso revertir, el fracaso de órgano; sin embargo, aunque muchos clínicos han instituido el tratamiento mantenido como terapia standard y especialmente en enfermos diabéticos (Desouza et al 2002) ${ }^{11}$, escasean aún los trabajos que definitivamente confirmen esta hipótesis y más aún, la extiendan al resto de las estructuras endoteliales del cuerpo humano. Sorprende que dosis de $5 \mathrm{mg}$ de Tadalafil tengan igual efecto que dosis de $10 \mathrm{mg}$. (Porst et al 2006) ${ }^{15}$. Se ignora el papel de la taquifilaxia, al menos hasta ahora, en tratamientos de larga duración.

Se especula sobre el mecanismo intimo de la "potencial curación" de la disfunción endotelial con el tratamiento continuado; la D.E. que sigue a la prostatectomía radical es un modelo de estu- 
Tabla 3

Ensayos clínicos más recientes con tratamiento continuado, pautado o crónico

\begin{tabular}{|c|c|c|c|}
\hline Autor y año & Producto. Dosis. Pts. & Modelo de estudio & Conclusiones y comentarios \\
\hline Desouza et al 2002 & Sildenafil $25 \mathrm{mg}$ & $\begin{array}{l}\text { Diabetes tipo II, doble ciego controlado } \\
\text { con placebo y cruzado }\end{array}$ & Mejora flujo arteria braquial \\
\hline Sommer et al 2005 & $\begin{array}{l}\text { Sildenafil } 50 \mathrm{mg} / \\
\text { cada noche }\end{array}$ & & $\begin{array}{l}\text { Mejoría significativa que se mantiene } \\
\text { al suspender la medicación. }\end{array}$ \\
\hline Caretta et al 2005 & $\begin{array}{l}\text { Tadalafil } 20 \mathrm{mg} \text { dias } \\
\text { alternos x } 3 \text { meses }\end{array}$ & Pacientes entre $60-70$ años & Respuesta satisfactoria en curaciones \\
\hline $\begin{array}{l}\text { MacMahon, CG. } \\
\text { 2004-2005 }\end{array}$ & Tadalafil $10-20 \mathrm{mg}$ & $\begin{array}{l}\text { Pacientes sin respuesta tratamiento a } \\
\text { demanda }\end{array}$ & $\begin{array}{l}\text { Buena respuesta a tratamiento } \\
\text { continuado. Preferencia por la dosis } \\
\text { de } 20 \mathrm{mg} \text {. }\end{array}$ \\
\hline Mirone et al 2005 & $\begin{array}{l}\text { Tadalafil } 20 \text { mg- } 3 \\
20 \text { mg., a demanda x } \\
\text { s vs } 20 \text { mg. } \\
3 \text { veces x semana x } \\
5-6 \text { semanas }\end{array}$ & $\begin{array}{l}\text { Multicéntrico cruzado y abierto } \\
\text { explorando preferencias de los pacientes }\end{array}$ & $\begin{array}{l}\text { Mejores resultados con tratamiento } \\
\text { continuado. Pacientes prefirieron } \\
\text { régimen a demanda (58\%) }\end{array}$ \\
\hline Porst et al 2006 & $\begin{array}{l}\text { Tadalafil } 5 \text { y } 10 \text { mg. } x \\
12 \text { semanas, } 268 \text { pacientes }\end{array}$ & $\begin{array}{l}\text { Investigan efectos dosis diferentes, } \\
\text { eficacia con } 10 \mathrm{mg}\end{array}$ & $\begin{array}{l}\text { Seguramente } 5 \mathrm{mg} \text {. suficiente, } \\
\text { similar } 10 \mathrm{mg}\end{array}$ \\
\hline
\end{tabular}

dio interesante que ha motivado investigaciones para prevenirla (Montorsi et al 1997) ${ }^{16}$ acelerar su recuperación (Montorsi et al 200014, PadmaNathan et al $2003^{3}$, Mullhall et al 2005 ${ }^{20}$ ), e incluso indagar sobre los mecanismos de rehabilitación. Se considera que intervienen prioritariamente al menos dos factores en este proceso; la oxigenación mantenida (Moreland et al 2001 ${ }^{3}$, Schwartz et al $2004^{21}$ ) y el incremento de células progenitoras circulantes (Foresta et al 2005 22 ). En estas circunstancias el objetivo fundamental de la rehabilitación peneana, en este caso exclusivamente peneana, es evitar el daño del tejido cavernoso que ocurre durante el periodo de recuperación neural suministrando la oxigenación necesaria. La oxigenación produce un aumento de los prostanoides intracavernosos necesarios para la protección del músculo liso cavernoso, tanto en el aspecto funcional como estructural. En este sentido el estudio de Schwartz et al (2004) ${ }^{21}$, ha demostrado que el uso mantenido de 50 o 100 mg. de Sildenafil, en dosis nocturnas y durante seis meses después de la prostatectomía radical con preservación de las bandeletas neurovasculares, preserva, en cantidades relacionadas con la dosis, el músculo liso cavernoso. El aumento de células progenitoras circulantes, en experiencias humanas con vardenafil, es una comproba- ción importante ya que estas células juegan un papel relevante en el proceso de neovascularización y contínua remodelación del endotelio; por otro lado, su reducción está asociada con el incremento de factores de riesgo cardiovascular. Otros factores que pueden participar en la rehabilitación peneana son los siguientes: promoción de la neurogénesis, protección del endotelio cavernoso; actividad antifibrinogénica; angiogénesis; crecimiento del músculo liso cavernoso y diferenciación del mismo (Burnett, A.L. 200523).

\section{INSTRUMENTOS DE VALORACIÓN DE EFICACIA: E1 PAPEL DEL LABORATORIO}

Un tema que merece especial atención está relacionado con la validación de los instrumentos empleados para estos estudios, fundamentalmente cuestionarios con baremos comparativos a lo largo del tratamiento. El objetivo fundamental ha sido conocer la eficacia y tolerancia del fármaco comparando cambios del Indice Internacional de Función Eréctil y el Diario de Encuentros Sexuales (preguntas 2 y 3; penetración y terminación satisfactoria). Sólo recientemente se ha estimado valorar si el tratamiento continuado "mejora progresivamente" la función eréctil, y lo que es más orientador, cuántas "curaciones” de la disfunción eréctil (baremo 26-30) se producen y 
cuántos pacientes mantuvieron una función eréctil satisfactoria cuando se suspendió el tratamiento, y durante cuanto tiempo. Sólo ocasionalmente se han empleado métodos para valorar la respuesta sistémica endotelial, como el test del incremento del flujo tras la compresión braquial, el grosor de la arteria carotídea, eco-doppler peneano, y otros (Kaiser et al $2004^{24}$, Boicchio et al $2005^{25}$, Katz et al $2000^{26}$, Dishy et al $2001^{27}$ y Gori et al 2005 28 ).

Un aspecto en el que se reconocen graves deficiencias está relacionado con las técnicas de laboratorio empleadas para valorar la potencial mejoría endotelial conseguida con los tratamientos continuados. De hecho en la mayoría de los trabajos citados no se menciona ninguna técnica de laboratorio que incluya marcadores endoteliales y su respuesta al tratamiento. Bocchio et al $2004^{25}$ han estudiado marcadores bioquímicos de activación endotelial, considerados como iniciadores de la ateroesclerosis. Comparando pacientes con D.E. asociada a factores de riesgo vascular y otro grupo sin factores de riesgo, investigaron marcadores bioquímicos y estructurales y entre ellos; el espesor de la intima media carotídea, selectina P soluble, moléculas de adhesión intercelular (ICAM)-1, moléculas de adhesión vascular (VCAM)- 1 y endotelina 1 . Los resultados revelaron que no existían diferencias en cuanto al espesor de la intima media carotídea entre ambos grupos, pero si se encontró que los marcadores bioquímicos señalados eran significativamente más altos en los pacientes con D.E. sin riesgos vasculares que en los pacientes con riesgos cardiovasculares. Con estos resultados concluyen que la D.E. es un marcador temprano de arterioesclerosis y que los síntomas de D.E. pueden preceder cambios orgánicos, estructurales como la obstrucción de grandes o pequeños vasos, una teoría más ampliamente desarrollada por Montorsi et al 200026).

A juzgar por la información de la literatura actual, el único proyecto en el que se ha tomado como referencia fundamental los cambios que produce el tratamiento continuado en marcadores de función endotelial, es nuestro proyecto, aún inconcluso, pero con evidencias previas de clara utilidad de la fracción $\beta_{1}$ de la guanilatociclasa (García Cardoso et al. 2004²9, López Farré et al. 2006) ${ }^{30}$. Inicialmente se confirmo que los cambios que suceden en el endotelio cavernoso y músculo trabecular pueden ser investigados en células mononucleares de sangre periférica, lo que facilita de manera considerable el estudio de estos pacientes, soslayando punciones y aspiraciones de los cuerpos cavernosos o biopsias del músculo cavernoso. Se comprobaron más tarde, las peculiaridades de la disfunción endotelial que se producen en la vía del oxido nítrico, reconociéndose, en comparaciones con controles sanos, un notabilísimo incremento, estadísticamente significativo, de la fracción $\beta_{1}$ de la guanilatociclasa y de la sintasa del oxido nítrico, pareciendo el incremento de la $\beta_{1}$ guanilatociclasa como marcador más específico. Actualmente, en un estudio aún inconcluso, en el que se utiliza 100 mg de Sildenafil dos días a la semana durante un periodo de seis meses, se ha podido confirmar que existe una clara tendencia al incremento de la $\beta_{1}$ guanilatociclasa en pacientes tratados de manera continuada, mucho más notable que en los pacientes tratados a demanda.

Aunque citados, y circunstancialmente evaluados, hay otros marcadores de disfunción endotelial, pero se carece de evidencias sobre su aplicabilidad clínica.

Tampoco se han investigado niveles plasmáticos de PDE-5 en variadas circunstancias clínicas y sus modificaciones, sobre todo a largo término, cuando se usa uno u otro régimen. La larga vida media de Tadalafil $(17,5$ h.) y el resultante periodo de $36 \mathrm{~h}$ de respuesta, ha sido invocado como el perfil ideal para el tratamiento crónico. Con una dosis entre 2.5-20 mg el incremento de los niveles plasmáticos de Tadalafil es proporcional a la dosis consiguiéndose concentraciones plasmáticas estables a partir del quinto día y que superan al menos en 1.6 veces a la que se consigue con una sola dosis. Sin embargo, no parece existir una correlación clara entre niveles plasmáticos de PDE-5 y respuesta clínica ya que se han conseguido resultados similares con la dosis de 5-10 mg. (Forge et al 2006 ${ }^{31}$ ). En nuestra experiencia los niveles plasmáticos de PDE-5 se incrementaron de manera significativa tras 6 meses de tratamiento reduciéndose durante la fase de placebo a cifras inferiores a las basales. En los pacientes en el régimen a demanda, los niveles de 
PDE-5 han sido inferiores a los basales, sin que podamos encontrar una explicación razonable a este hallazgo.

\section{ESTUDIOS EN ANIMALES}

En la investigación animal se han buscado contestaciones concretas a diversos problemas, empleando tanto técnicas "in vitro" como "in vivo". Ha podido demostrarse que el tratamiento continuado incrementa la respuesta a los estímulos productores de oxido nítrico.

Diversos estudios han producido, hasta el momento, evidencias directas de que los PDE-5 pueden mejorar la estructura y funcionalidad del tejido cavernoso, sugiriendo, simultáneamente, los mecanismos moleculares implicados. De Young et al $\left(2003^{32}\right)$ ha podido demostrar en ratas diabéticas que la dosis de $5 \mathrm{mg} \mathrm{x} \mathrm{kg}$ de Sildenafil oral, en administración diaria, durante 3 semanas, preserva la morfología del tejido peneano, la fisiología de la erección y la sintasa del oxido nítrico neural más que el placebo.

Behr-Roussel et al $\left(2005^{33}\right)$ administró durante 8 semanas consecutivas una dosis de 60 $\mathrm{mg} / \mathrm{kg}$ por vía subcutánea a ratas, confirmando, en baño de órgano, que tiras de tejido cavernoso tenían una mejor respuesta al estímulo con acetilcolina que el mismo grupo de ratas no tratadas; sin embargo, no respondían a calcio o nitroprusiato sódico.

Musicki et al $2005^{34}$ comparó la respuesta de ratas jóvenes y viejas al estímulo con $20 \mathrm{mg} / \mathrm{kg}$ de Sildenafil aplicados por vía subcutánea cada 8 horas durante tres semanas, al mismo tiempo que se determinaron niveles plasmáticos, confirmando que las ratas viejas conseguían mejoría en la habilidad de la erección e incremento de la sintasa del oxido nítrico endotelial, un marcador que se ha asociado con incremento del flujo al tejido cavernoso. No se consiguieron los mismos efectos en ratas jóvenes por lo que se concluyó que en condiciones normales estos estímulos pueden ser contrarrestados por la activación del sistema rhoquinasa que limita la función eréctil preservando el riesgo de priapismo.

En general, la investigación animal ha demostrado que el uso continuado de los IPDE-5 ejerce un efecto saludable sobre el tejido eréctil con beneficios mantenidos.

\section{CONCLUSIONES}

El tratamiento continuado con los inhibidores de la PDE-5 constituye una estrategia terapéutica enormemente atractiva por sus potenciales efectos curativos sobre la disfunción eréctil en primer lugar, y la disfunción endotelial en segundo término. De no alcanzarse estos objetivos el concepto de prevención del proceso degenerativo endotelial ha sido también invocado. Sin embargo, las evidencias clínicas aportadas por los tratamientos a largo plazo, actualmente entre 4 y 6 meses, han conseguido confirmar algunos aspectos logísticos como la preferencia del enfermo, la mejoría mantenida de la D.E., etc., pero la hipótesis de la "curación" no ha sido confirmada; cuando se suspende el tratamiento recidiva la D.E. y desaparecen los potenciales beneficios sobre la enfermedad endotelial sistémica. Es este, por lo tanto, un proyecto inacabado que requerirá aún muchos años de investigación. Por el contrario, el objetivo podría facilitarse de manera notable si se observaran cambios significativos en marcadores bioquímicos de disfunción endotelial, tanto a corto como largo plazo. Desgraciadamente estos marcadores están aún en fase de identificación y las evidencias de los comúnmente empleados, incluidos los estudiados en nuestro laboratorio, no han podido hasta ahora mostrar su solidez y validez científica.

\section{REFERENCIAS}

1. McMahon C. Efficacy and safety daily tadalafil in men with erectile dysfunction previously unresponsive to on-demand tadalafil. J Sex Med. 2004;1(3):292-300.

2. Bivalacqua TJ, Usta MF, Champion HC, Kadowitz OJ, Hellstrom WJ. Endothelial dysfunction in erectile dysfunction: role of the endothelium in erectile physiology and disease. J Androl. 2003; 24(Suppl 6):17-37.

3. Moreland, RB. Pathophysiology of erectile dysfunction: the contributions of trabecular structure to function and the role of functional antagonism. Int $J$ Impot Res. 2000;12(Supple 4):S39-46. 4. Goldstein I. Chronic dosing of PDE-5 inhibitors should become the norm as such treatment improves erectile, heart and endothelial health European Society for sexual medicine. Viena, Diciembre 2. 2006.

5. Padma-Nathan E, McCullough AR,Giuliano F,Toler SM. Wohlhuter C, Shpilsky AB. Postoperative nightly administration of sildenafil citrate significantly improves the return of normal spontaneous erectile function after bilateral nervesparing radical prostatectomy, J Urol. 4(2003); (Suppl.), p. 375 .

6. Rosen R, Altwein J, Boyle P et al. Lower Urinary tract symptoms and male sexual dysfunction: tje multinational survey, of the aging male (MSAM-7) Eur Urol. 2003;44(6):637-649. 
7. McMahon C. Efficacy and safety daily tadalafil in men with erectile dysfunction previously unresponsive to ondemand tadalafil. J Sex Med. 2004;1(3):292-300.

8. McMahon C. Comparison of efficacy, safety and tolerability of on-demand tadalafil and daily dosed tadalafil for the treatment of erectile dysfunction. J Sex Med. 2005;2(3):415-427

9. Mirone, $\mathrm{V}$, van Ahlen, $\mathrm{H}$ and for the SURE Study Investigators. An evaluation of an alternative dosing regimen with tadalafil, 3 times/week, for men with erectile dysfunction: SURE study in 14 European countries., Eur Urol. 2005 Jun;47(6):846-54; discussion 854. Epub 2005 Mar 9.

10. Martin Morales, A, Moncada Iribarren, I, Cruz Navarro, N, Sanz Terrada, B, Casinello Hervas, A, Chan, M, Casariego García-Luben, J. Eficacia y seguridad de Tadanafilo en dos regímenes de dosificación diferentes en pacientes españoles con disfunción eréctil: Resultados procedentes del Estudio SURE realizado en 14 pacientes europeos. Actas Urol. Esp. 2006:30(8): 791-800.

11. Desouza C,Parulkar A,Lumpkin D, Akers D, Fonseca V. Acute and prolonged effects of sildenafil on brachial artery flow-mediated dilatation in type 2 diabetes. Diabetes Care 25 (2002), pp. 1336-1339

12. Sommer F, Schulze WTreating erecyile dysfunction by endothelial rehabilitation with phosphodiesterase 5 inhibitors. World J Urol. 2005; 23(6):385-392

13. Rosano GM, Aversa A, Vitale C, Fabbri A Finin M, Spera G. Chronic treatment with tadalafil improves endothelial function in men with increased cardiovascular risk factors. Eur Urol. 2005 Feb;47(2):214-220

14. Caretta N, Palego P, Ferlin A, Garolla A, Bettella A, Selice R, Foresta C. Resumption of spontaneous erections in selected patients affected by erectile dysfunction and various degrees of carotid wall alteration: Role of tadalafil, Eur Urol 48 (2005), pp. 326-332.

15. Porst H, Giuliano F, Glina S, Ralph D, Casabe AR.ElionMboussa, A, Shen, W, Whitaker, JS.: Evaluation of the efficacy abd safety of Once-a-Day dosing of Tadalafil $5 \mathrm{mg}$ and $10 \mathrm{mg}$ in the treatment of erectile dysfunction: Results of a Multicenter, Randomized, Double-blind, placebo-controlled trial. Europ Urol. 50(2) 2006. 351-359.

16. Montorsi F, Briganti A, Salonia A, Rigati P, Burnett AL. Can phosphodiesterase type 5 inhibitors cure erectile dysfunctionß. Europ Urol. 2006 (49) 979-986

17. Hellstrom J.W..G, Kendirci, M, Type 5 phosphodiesterase inhibitors: Curing erectile dysfunction. Eur Urol. 2006 Jun;49(6):942-945

18. Montorsi F, Guazzoni G, Strambi L.F, Da Pozzo L.F, Nava L, Barbieri, L. et al. Recovery of spontaneous erectile function after nerve-sparing radical retropubic prostatectomy with and without early intracavernous injections of alprostadil: results of a prospective, randomized trial. $\mathrm{J}$ Urol. 1997;158(4): 1408-1410.

19. Montorsi F, Salonia A, Barbieri L, Maga T, Zanoni M, Raber M. et al., The subsequent use of I.C. alprostadil and oral sildenafil is more efficacious than sildenafil alone in nerve sparing radical prostatectomy patients, (abstract). J Urol 167 (2002) (Suppl.)p. 279.

20. Mulhall J, Land S, Parker M, Waters WB, Flanigan R.C.The use of an erectogenic pharmacotherapy regimen following radical prostatectomy improves recovery of spontaneous erectile function. J Sex Med. 2005;2(4):532-540

21. Schwartz E, J, Wong, E.J. and Graydon, R.J.: Sildenafil preserves intracorporeal smooth muscle after radical retropubic prostatectomy, J Urol. 2004 Feb;171(2 Pt 1):771-774.
22. Foresta C, Lana A, Cabrelle A, et al: PDE-5 inhibitor, Vardenafil, increases circulating progenitor cells in humans. Int J Impot Res. 2005;17(4):377-380.

23. Burnett AL. Erectile dysfunction following radical prostatectomy, JAMA. 2005;293(21):2648-2653.

24. Kaiser DR, Billups K, Mason C, Wetterling R, Lundberg JL. Bank AJ. Impaired brachial artery enddotehlium-dependent and independent vasodilation in men with erectile dysfunction and no other clinical cardiovascular disease, $\mathrm{J}$ Am Coll Cardiol. 2004; 43(2):179-84.

25. Bocchio M, Desideri G, Scarpelli P. Necozione S, Properzi G, Spartera C, et al: "Endothelial cell activation in men with erectile dysfunction without cardiovascular risk factors and overt vascular damage. J Urol. 004;171(4):1601-1604.

26. Katz SD, Balidemaj K, Homma,S, Wu H, Wang, J, Maybaum S. Acute type 5 phosphodiesterase inhibition with sildenafil enhances flow-mediated vasodilation in patients with chronic heart failure, J Am Coll Cardiol 36 (2000), pp. 845-851.

27. Dishy V, Sofowora G, Harris PA, Kandcer M, Zhan F, Wood AJ, Stein CM. The effect of sildenafil on nitric oxidemediated vasodilation in healthy men, Clin Clin Pharmacol Ther. 2001;70(3):270-9

28. Gori T, Sicuro S, Dragoni S, Donati G, Forconi S. Parker JD. Sildenafil prevents endothelial dysfunction induced by ischemia and reperfusion via opening of adenosine triphosphate-sensitive potassium channels, Circulation. 2005 Feb 15;111(6):721-3.

29. Garcia Cardoso, JV, Lopez Farre, A, Vela Navarrete, R. Disfunción eréctil. Papel del Laboratorio en la evaluación diagnóstica y pronóstica. Actas Urol Esp. 2005; 29(9):890897.

30. Mateos Caceres J, Garcia Cardos JV, Lapuente L, Zamorano León D, Sacristan T, de Prada P, Calahorra,F, Macaya, C, Vela Navarrete. Soluble Guanylate Cyclase $\beta$ 1subunit expression is increased in mononuclear cells from patients with erectile dysfunction. Int $\mathrm{J}$ Impot Res, Epub March 9. 2006.

31. Forgue ST, Patterson BE, Bedding AW, Payne CD, Phillips DL, Wrishko RE, Mitchell MI. Tadalafil pharmacokinetics in healthy subjects. Br J Clin Pharmacol. 2006; 61(3): 280-288.

32. De Young, D. Yu, D. Freeman and G.B. Brock, Effect of PDE5 inhibition combined with free oxygen radical scavenger therapy on erectile function in a diabetic animal model. Int J Impot Res. 2003):15(5):347-354.

33. Behr-Roussel D, Gorny D, Mevel K, Caisey S, Bernabe J, Burgess G, Wayman C,.et al., Chronic sildenafil improves erectile function and endothelium-dependent cavernosal relaxations in rats: lack of tachyphylaxis. Eur Urol. 2005; 47(1):87-91.

34. Musicki B, Champion HC, Becker RE, Liu T, Kramer MF. Burnett AL. Erection capability is potentiated by long-term sildenafil treatment: role of blood flow-induced endothelial nitric-oxide synthase phosphorylation, Mol Pharmacol. 2005;68(1):226-232.

Correspondencia autor: Dr. R. Vela Navarrete

Servicio de Urología. Fundación Jiménez Díaz.

Avda. de los Reyes Católicos, 2. 28040 Madrid

Tel.: 915504800

E-mail autor: rvelan@telefonica.net

Información artículo: Editorial

Trabajo recibido: enero 2007

Trabajo aceptado: febrero 2007 